\title{
ANGULAR RATE SENSING BY CIRCULATORY VORTEX FLOW: DESIGN, SIMULATION AND EXPERIMENT
}

\author{
Hoa Thanh Phan ${ }^{1 *}$, Thien Xuan Dinh ${ }^{2}$, Canh-Dung Tran ${ }^{3}$, Trinh Chu Duc ${ }^{4}$,Tung Thanh Bui ${ }^{4}$, \\ Phuc Hong Pham ${ }^{5}$, and Van Thanh Dau \\ ${ }^{1}$ Hanoi University of Industry HaUI, HaUI Institute of Technology, VIETNAM \\ ${ }^{2}$ Graduate School of Science and Engineering, Ritsumeikan University, JAPAN \\ ${ }^{3}$ School of Mechanical and Electrical Engineering, University of Southern Queensland, \\ AUSTRALIA \\ ${ }^{4}$ VNU University of Engineering and Technology, Hanoi, VIETNAM \\ ${ }^{5}$ School of Mechanical Engineering, Hanoi University of Science and Technology, VIETNAM \\ ${ }^{6}$ School of Engineering and Built Environment, Griffith University, AUSTRALIA
}

\begin{abstract}
A fully packaged convective vortex gyrometer actuated by a PZT diaphragm is reported. The flow circulates at higher velocity after each actuating circle to form a vortex in the desired chamber. The vortex is characterized by hotwire anemometry. The device is initially designed based on a numerical analysis whose results are used to set up the experiment. The angular rate sensing of the device is successfully tested using a turntable. The technique is a potential solution to various applications related to inertial sensing and fluidic amplifier.
\end{abstract}

\section{KEYWORDS}

Vortex, synthetic jet, gyroscope, PZT diaphragm, COMSOL

\section{INTRODUCTION}

Vortex-based inertial fluidic systems presented in several recent publications include vortex based inertial fluidic system and fluidic amplifier [1]-[3] and vortex based gyrometer [4]. While fluidic gyroscopes are typically categorized as jet flow gyroscope and thermal gas gyroscope with sufficient literature reports on laminar gas stream, corona discharge or thermal expansion principles [5]-[12], there has not been any adequate technical information disclosed for vortex gyrometer. More recently, some groups studied multi-axis inertial sensor using the vortex movement; nevertheless, the mechanism was not sufficiently described [13], [14]. From our understanding, besides the use of an external pump to create vortex flows, which is bulky and expensive, the other techniques have not been adequately presented. Thus, a self-package vortex based gyrometer is reported here.

The flow is synthesized by an actuator that consists of a cavity sealed at one end and emitting nozzles at the other end. This technique is usually mentioned as synthetic jet where the flow is rectified by means of a train of vortices behind the edges of the nozzle [15]-[20]. For the present approach, a conventional PZT diaphragm is used to actuate a circulating flow inside a closed system. With the vibration of the PZT diagram, air flow moving through a rectifying nozzle creates a small net flow in driving channels at each cycle [21]. In other words, the process by the PZT diagram vibration generates a synthetic jet of a net flow which propagates by a gradual circulation toward a feedback chamber and then backward the vortex chamber. Furthermore, a vortex flow generated in the system by the circulating flow whose velocity which can be controlled by the vibration magnitude of the PZT is investigated by both the simulation and experiment. A unique advantage of the synthetic jet compared with the conventional vortex approaches is that it can be entirely generated and developed by the air flow inside a system. Hence, synthetic jets can transfer linear momentum to the flow without any mass injection from outside over a broad range of length and timescale [22]. Thus, this approach is potential in several applications related to fluidic actuator for controlled flows.

\section{VORTEX FLOW-BASED SENSOR AND SIMULATION METHOD}

The sensor configuration in Fig. 1 a includes a vibrating PZT diaphragm to move air through four rectifying nozzles to form small net flows in driving channels at each cycle. This process creates four synthetic jet flows which gradually propagates toward the chamber where their tangent movements create a clockwise vortex. At the center of this chamber, the vortex sinks and leave the chamber before being rectified again toward the driving channels. The design of this sensor comprises a disc-cylinder with diameter of $20 \mathrm{~mm}$ and $5.5 \mathrm{~mm}$ in length with a pump chamber in one side and a vortex space on the opposite side. This vortex cavity connects to pump chamber by four driving channels with a diameter of $1.5 \mathrm{~mm}$ each at the outermost edge of the cylinder. At the center of the vortex chamber, the feedback channel with a diameter of $3 \mathrm{~mm}$ linked back to four driving channels to form a rectifying nozzle.

The pump chamber works by the vibration of PZT diaphragm under an applied voltage, volume of the pump chamber is shrinking and swelling due to PZT vibration. The air inside the chamber is alternatively expelled and sucked in each vibration cycle. Due to the rectification of the nozzle, a net flow is generated inside driving channels in each cycle. This small net flow travels into the vortex cavity, circulates and then moves back the rectifying nozzle via a feedback channel. The circulating flow together with its momentum dramatically amplifies the rectifying effect of the nozzle. After certain circulations, the velocity and also the momentum of flow reach values enough high to generate a vortex inside the vortex chamber. 

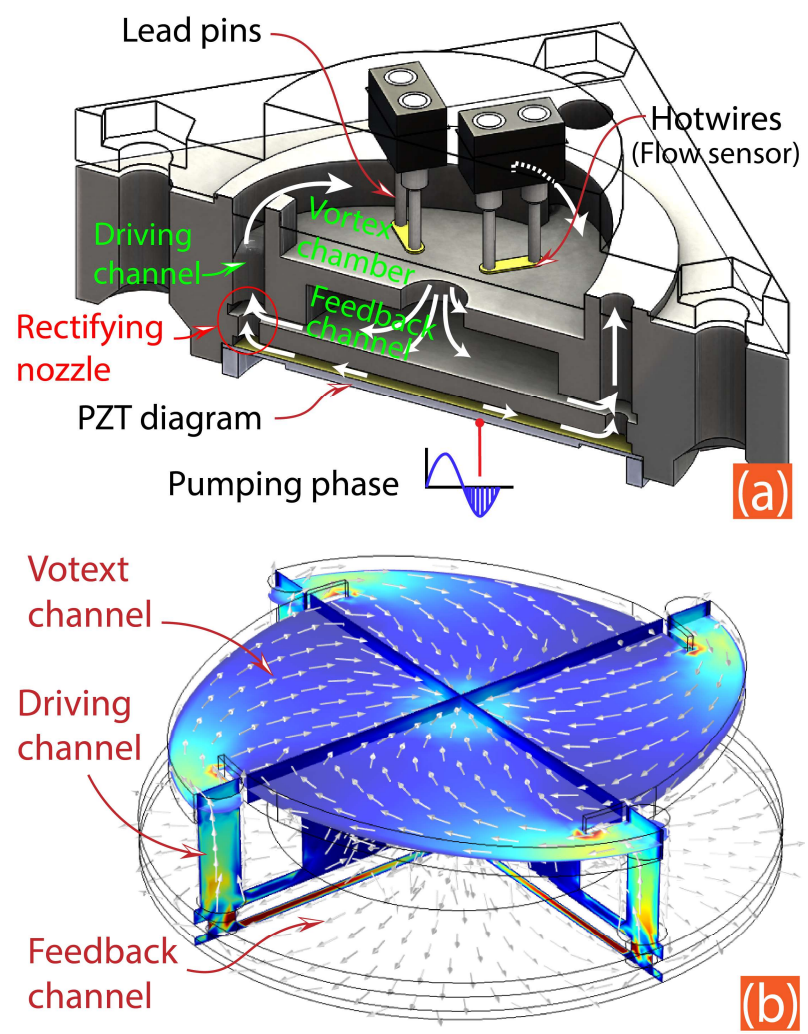

Figure 1: (a) Design, mechanism of vortex generator and (b) Meshing for simulation.

The equations to calculate the circulating flow in channels is shown as bellow:

$$
\begin{aligned}
& \frac{\partial \rho}{\partial t}+\nabla \cdot \rho \vec{u}=0 \\
& \frac{\partial \rho^{\vec{u}}}{\partial t}+(\vec{u} \cdot \nabla) \rho \vec{u}=-\nabla p+\nabla \cdot(\mu \nabla \vec{u})-2 \rho \vec{\omega} \times \vec{U}(2) \\
& \frac{\partial \rho_{p} T}{\partial t}+(\vec{u} \cdot \nabla) \rho c_{p} T=\nabla \cdot(\lambda \nabla T)
\end{aligned}
$$

Where term $2 \rho \vec{\omega} \times \vec{U}$ represents the Coriolis apparent acceleration in a rotating frame with the angular velocity $\vec{\omega} ; \vec{u}, p$, and $T$ denote the velocity vector, pressure, and temperature of the flow field, respectively; $\mu=1.789 \times$ $10^{-5}$ Pas, $\quad \rho=1.2041 \mathrm{kgm}^{-3}, \quad \lambda=2.42 \times$ $10^{-3} \mathrm{Wm}^{-1} \mathrm{~K}^{-1}$, and $c_{p}=1006.43 \mathrm{Jkg}^{-1} \mathrm{~K}^{-1}$ are the dynamic viscosity, density, thermal conductivity, and specific heat of gas, respectively. Since the working gas is air, the relationship between the pressure and density follows the state equation of an ideal gas $p=\rho R_{u} T / M_{w}$, where $R_{u}=8.314 \mathrm{Jmol}^{-1} \mathrm{~K}^{-1}$ is the universal air constant and $M_{w}=28.96 \mathrm{gmol}^{-1}$ the molecular weight.

The boundary condition imposed on the diaphragm is derived from its vibrating rate $v(\vec{r}, t)=$ $2 \pi f Z \cos (2 \pi f t) \varphi(\vec{r})$ with the shape function $\varphi(r)=$ $\left(1-(r / a)^{2}\right)^{2}$, where $a$ is the diaphragm radius and $Z$ the center deflection of the PZT diaphragm. The transient solution is obtained by our program code developed in the environment OpenFOAM.

The mechanism illustrated by the simulated flow through meshing sensor structure is presented in Fig. $1 \mathrm{~b}$ where the PZT diaphragm vibration is used as the boundary condition for the 3D-transient analysis.

\section{RESULTS AND DISCUSSION Simulation results}

For simulation work, the working principle of sensor based on the vortex flow is indicated in Fig. 2. Figure 2(a) shows that under an angular rate $\omega_{z}$ about Z-axis, the appearing vortex is deflected along the radial direction by the Coriolis' effect. Figure 2(b) presents the distribution of simulated flow velocity under the effect of angular rate. For details, Figure 2(c) plots the evolution of flow velocity at a test point $(0,5,5)$ near the edge of the vortex chamber over 30 vibrating cycles of PZT diaphragm with and without angular rate. Simulation results show clearly the difference in the velocity at the test point with an applied angular rate.
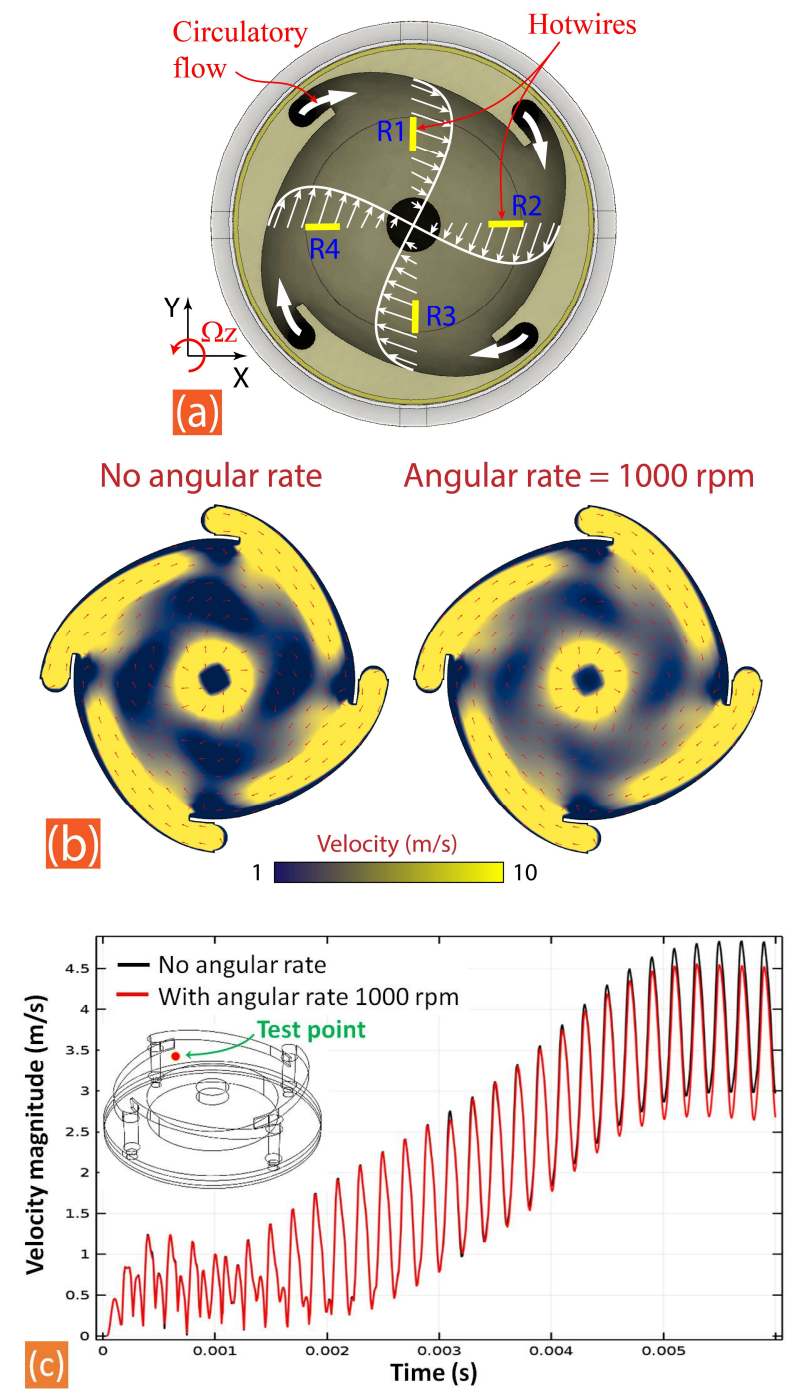

Figure 2: Numerical simulation: (a) Sketch showing working principle; (b) Effect of angular rate on vortex confirmed by simulation and (c) The transient velocity of flow at the test point $(0,5,5)$ over 30 PZT vibration cycles for two cases without applied angular rate and with angular rate applied from $15^{\text {th }}$ cycle (i.e. from $0.003 \mathrm{~s}$ )

In order to show more detail of vortex flow travelling inside the vortex chamber of sensor, we use high-speed camera to capture the trajectory movement of particles in the vortex cavity. A prototype of the system made of polymethyl methacrylate with a transparent cover and a highspeed camera was set up to capture the motion of particles 
as shown in Fig.3(a). Up to the voltage applied on PZT diaphragm reached $20 \mathrm{~V}$, a vortex appears in the chamber, which is in a good agreement with the simulation.

\section{Experimental achievements}

The above-simulated results were then investigated by the experiments for the vortex-based sensor with tungsten hotwires installed inside the vortex chamber placed on the turntable. The experiment setup is described in Fig.3(b). The rotation frame in the simulation is tested by using a self-developed turntable, whose angular velocity is monitored by a direct current motor with an integrated encoder (Tsukasa Electric Ltd.). The on-chip circuit is slipring connected to the off-chip circuit to transfer signal while the turntable disk rotates. For time-resolved analysis, the experimental data are continuously recorded and monitored by a computer system using the NI Signal Express-data (National Instrument Ltd.) logging software connected to NI9234 data acquisition device with a sampling rate of $25.6 \mathrm{kHz}$. The output signal is measured by the output voltage on the hotwires.

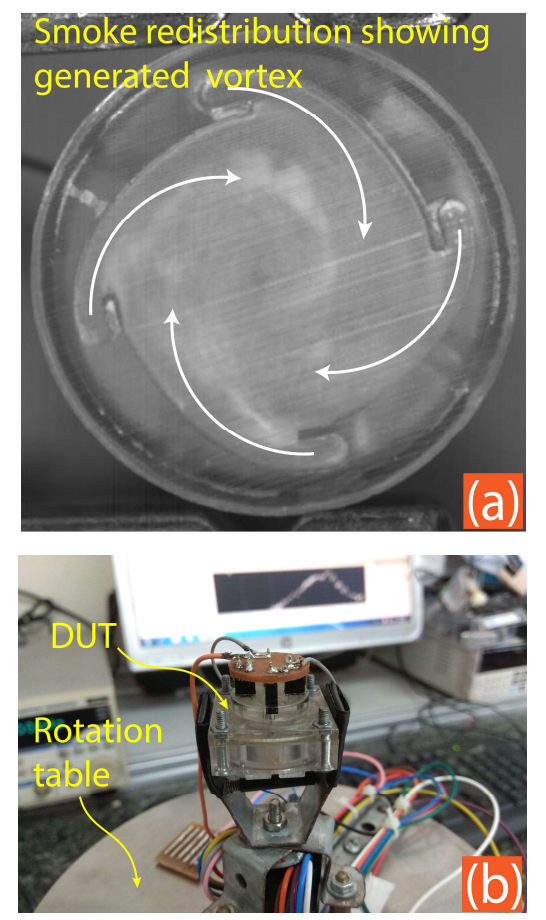

Figure 3: Experimental work. (a) Fabricated prototype with a vortex observed by high-speed camera; and (b) Experimental setup showing the angular rate sensing measurement with a turntable.

With a specified voltage of $50 \mathrm{~V}$ applied on the PZT and hotwires heated by a current of $200 \mathrm{~mA}$, the experimental results, as presented in Fig.4(a), demonstrate an increase of the output voltage on the hotwire with an increase of the actuating frequency of the PZT. The output voltage decreases rapidly after reaching its peak at a frequency of $4.67 \mathrm{kHz}$. In addition, Figure 4(b) indicate the relationship between the output voltage on hotwire $V_{H W}$ and driving voltage of the PZT $\left(V_{P Z T}\right)$ while the system is actuating at a resonant frequency of $4.67 \mathrm{kHz}$ and heated by a current of $200 \mathrm{~mA}$. The result shows that the output voltage of the hotwire increases with the increase of the driving voltage of the PZT. This can be explained as a larger deformation of the diaphragm due to a stronger $V_{P Z T}$ produces a higher flow velocity [23].

In order to test the rotation measurement capability of the vortex-based sensor, the sensor is placed on the disk of the turntable at its center and its edge.
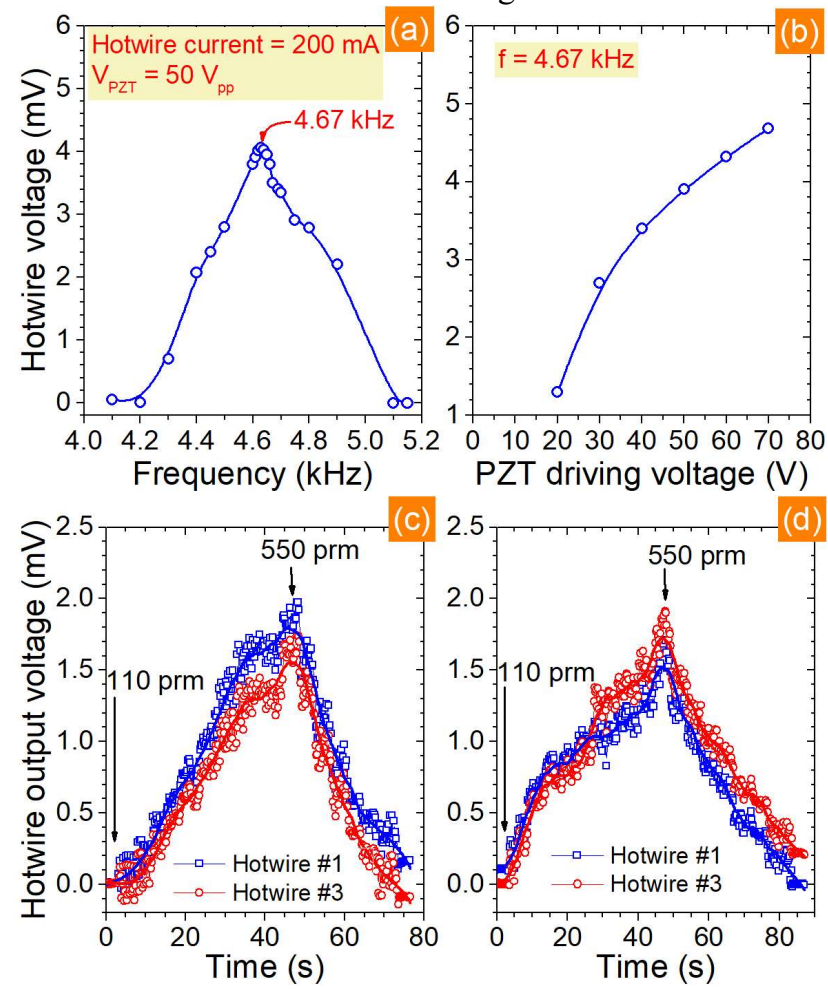

Figure 4: (Experimental results) Without rotation (a, b): hotwire voltage plotted versus (a) vibrating frequency of the PZT diaphragm (resonant frequency of $4.67 \mathrm{kHz}$ is recognized as the peak output of hotwire), (b) the PZT applied voltage using a driving frequency of $4.67 \mathrm{kHz}$; With rotation from $110 \mathrm{rpm}$ to $550 \mathrm{rpm}$ from turntable $(c, d)$ : (c) variation of hotwire voltage at when sensor is placed at the center and (d) at the edge of the turntable

Figure 4(c) shows that the hotwire voltage changes from $0.27 \mathrm{mV}$ to $1.88 \mathrm{mV}$ when the turntable rotates from $110 \mathrm{rpm}$ to $550 \mathrm{rpm}$. It is worth noting that although a small difference of output voltages on two hotwires $(<10 \%)$ their evolutions are the same. Additionally, the effect of centrifugal acceleration (linear acceleration) is tested by placing the sensor at the edge of the turntable, Figure 4(d) depicts that the additional linear acceleration only varies hotwire output voltage around $3.8 \%$.

\section{CONCLUSION}

In this paper, our prototype of sensor based-on a high velocity vortex flow with millimeter dimension is introduced and described in term of simulation and experiments. Vortex flow is generated in the sensing cavity when the flow actuated by a PZT diaphragm and its velocity increases after each circulation. The design of the device is firstly conducted by a simulation analysis and then its results are referred as the base of the experiment. Experimental results shown that the created vortex flow is 
deflected by the rotation that is indicated in different hotwire voltage levels. Moreover by observation of high speed camera, a flow vortex appears quite clearly. In the numerical evaluation as well as experimental achievements, it proves the potential of our sensor in numbers of applications related to inertial fluidic sensing.

\section{ACKNOWLEDGEMENTS}

This research is funded by the Vietnam National Foundation for Science and Technology Development (NAFOSTED) under grant number 107.01-2019.19.

\section{REFERENCES}

[1] Weathers and T. M., "NASA contributions to fluidic systems: A survey," Jan. 1972.

[2] G. L. Goglia and D. K. Patel, "An experimental and theoretical study of the flow phenomena within a vortex sink rate sensor," 1974.

[3] V. T. Dau, T. X. Dinh, T. T. Bui, and C. D. Tran, "Vortex flow generator utilizing synthetic jets by diaphragm vibration," Int. J. Mech. Sci., vol. 142143, no. May, pp. 432-439, 2018.

[4] T. Sarpkaya, "A pneumatic vortex angular rate sensor - analysis and experiments," Automatica, vol. 9, no. 1 (January, 1973), pp. 29-34, 1973.

[5] P. T. Hoa, T. X. Dinh, and V. T. Dau, "Design Study of Multidirectional Jet Flow for a Triple-Axis Fluidic Gyroscope," IEEE Sens. J., vol. 15, no. 7, pp. 41034113, Jul. 2015.

[6] G. Kock, P. Combette, B. Chariot, A. Giani, M. Schneider, and C. Gauthier-Blum, "Study and realization of a fluidic thermal gyrometer," in 2017 Symposium on Design, Test, Integration and Packaging of MEMS/MOEMS (DTIP), 2017, pp. 15.

[7] G. Kock, P. Combette, M. Tedjini, M. Schneider, C. Gauthier-Blum, and A. Giani, "Experimental and Numerical Study of a Thermal Expansion Gyroscope for Different Gases," Sensors, vol. 19, no. 2, p. 360, Jan. 2019.

[8] V. T. Dau, T. X. Dinh, C. D. Tran, T. T. Bui, and H. T. Phan, "A study of angular rate sensing by corona discharge ion wind," Sensors Actuators A Phys., vol. 277, pp. 169-180, Jul. 2018.

[9] V. T. Dau, T. X. Dinh, C. D. Tran, P. N. Bui, D. D. Vien, and H. T. Phan, "Fluidic mechanism for dualaxis gyroscope," Mech. Syst. Signal Process., vol. 108, pp. 73-87, 2018.

[10] T. X. Dinh, D. B. Lam, C. D. Tran, T. T. Bui, P. H. Pham, and V. T. Dau, "Jet flow in a circulatory miniaturized system using ion wind," Mechatronics, vol. 47, no. September, pp. 126-133, Nov. 2017.

[11] V. T. Dau, T. Shiozawa, D. V. Dao, H. Kumagai, and S. Sugiyama, "A dual axis gas gyroscope utilizing low-doped silicon thermistor," in 18th IEEE International Conference on Micro Electro Mechanical Systems, 2005. MEMS 2005., 2005, pp. 626-629.

[12] V. T. Dau, D. V. Dao, T. Shiozawa, and S. Sugiyama, "Convective Gas Gyroscope Based on Thermo-
Resistive Effect in $\mathrm{Si}$ P-N Junction," in TRANSDUCERS 2007 - 2007 International SolidState Sensors, Actuators and Microsystems Conference, 2007, pp. 2525-2528.

[13] H. Chang, P. Zhou, X. Gong, J. Xie, and W. Yuan, "Development of a tri-axis vortex convective gyroscope with suspended silicon thermistors," in 2013 IEEE SENSORS, 2013, pp. 1-4.

[14] H. Chang, P. Zhou, Z. Xie, X. Gong, Y. Yang, and W. Yuan, "Theoretical modeling for a six-DOF vortex inertial sensor and experimental verification," J. Microelectromechanical Syst., vol. 22, no. 5, pp. 1100-1108, Oct. 2013.

[15] N. Q. Dich, T. X. Dinh, P. H. Pham, and V. T. Dau, "Study of valveless electromagnetic micropump by volume-of-fluid and OpenFOAM," Jpn. J. Appl. Phys., vol. 057201, no. 5, p. 057201, May 2015.

[16] V. T. Dau, T. X. Dinh, Q. D. Nguyen, R. Amarasinghe, K. Tanaka, and S. Sugiyama, "Microfluidic valveless pump actuated by electromagnetic force," Proc. IEEE Sensors, no. Type II, pp. 679-682, 2009.

[17] T. X. Dinh, N. T. M. Le, V. T. Dau, and Y. Ogami, "A dynamic model for studying valveless electromagnetic micropumps," J. Micromechanics Microengineering, vol. 21, no. 2, p. 025015, Feb. 2011.

[18] H. Phan, T. Dinh, P. Bui, and V. Dau, "Transient Characteristics of a Fluidic Device for Circulatory Jet Flow," Sensors, vol. 18, no. 3, p. 849, Mar. 2018.

[19] K. Tanaka, V. T. Dau, R. Sakamoto, T. X. Dinh, D. V. Dao, and S. Sugiyama, "Fabrication and Basic Characterization of a Piezoelectric Valveless Micro Jet Pump," Jpn. J. Appl. Phys., vol. 47, no. 11, pp. 8615-8618, Nov. 2008

[20] V. T. Dau, T. X. Dinh, T. Katsuhiko, and S. Susumu, "A cross-junction channel valveless-micropump with PZT actuation," Microsyst. Technol., vol. 15, no. 7, pp. 1039-1044, May 2009.

[21] V. T. Dau, T. X. Dinh, and T. T. Bui, "Jet flow generation in a circulatory miniaturized system," Sensors Actuators B Chem., vol. 223, pp. 820-826, 2015.

[22] A. Glezer and M. Amitay, "Synthetic jets," Annu. Rev. Fluid Mech., vol. 34, no. 1, pp. 503-529, Jan. 2002.

[23] V. T. Dau and T. X. Dinh, "Numerical study and experimental validation of a valveless piezoelectric air blower for fluidic applications," Sensors Actuators B Chem., vol. 221, pp. 1077-1083, Jul. 2015.

\section{CONTACT}

*Hoa Thanh Phan, phanthanhhoa@haui.edu.vn 